Erschienen in: Stickel, Gerhard (Hrsg.): Sprache - Sprachwissenschaft - Öffentlichkeit. Berlin/New York: de Gruyter, 1999. S. 194-224.

(Jahrbuch des Instituts für Deutsche Sprache 1998)

DOI: https://doi.org/10.1515/9783110622645-014

HANS-WERNER EROMS

\title{
Die Rechtschreibreform in der öffentlichen Meinung
}

\begin{abstract}
Die geplante Rechtschreibreform der deutschsprachigen Länder hat eine überaus heftige Diskussion in der Öffentlichkeit hervorgerufen. Befüworter und Kritiker äußern sich in so gut wie allen kommunikativen Kanälen. Die seit Jahren geführte Debatte hat an Schärfe zugenommen und vielfach die sachlichen Bahnen verlassen. Das folgende Referat stützt sich auf die in den Printmedien geäußerten Ansichten und analysiert Berichte, Leserbriefe und Kommentare in ihrer Tendenz und ihrer Argumentationsstruktur. Dabei tritt zu Tage, daß in hohem Maße immer die gleichen Argumentationstopoi eingesetzt werden, allerdings mit unterschiedlicher Gewichtung. Aufs Ganze gesehen sind bei allem Engagement im einzelnen die Zeitungskommentare ausgewogener als vor allem die Leserbriefe, auch wenn die Argumentationsformen sehr ähnlich sind. Die Debatte um die Rechtschreibreform weist zunehmend Charakteristika der öffentlich-politischen Diskussion auf. Das Referat geht den Gründen dafür nach.
\end{abstract}

\section{Einleitung}

Seit nun fast drei Jahren ist die Rechtschreibreform des Deutschen ein festes Thema in den Zeitungs-, Hörfunk- und Fernsehredaktionen, auf Lehrerkonferenzen, in den Klassenzimmern und Seminarräumen und nicht zuletzt an den Stammtischen. Kaum eine kulturpolitische Thematik hat die Öffentlichkeit so erregt und so polarisiert wie die Änderung der Rechtschreibung und vor allem die Modalitäten ihrer Umsetzung. Wie gleich zu zeigen sein wird, tritt in der Öffentlichkeit die Sachdiskussion zurück, es geht von so gut wie allen Ansatzpunkten, die sich bei der Orthographie ergeben, häufig sogleich ins Grundsätzliche. Hier finden sich, wie bei den Debatten um Gegenstände der Politik, Bewertungen der Gegner, ,Aufklärung“ über die vermeintlich wahren Motive der Stellungnahmen für oder gegen die Reform, Belehrungen und Beschimpfungen, kurz alles das, was wir aus den politischen Debatten kennen, auch mit dem gleichen Effekt, vor allem mit dem der Abstumpfung und - in der jüngsten Zeit - dem des Überdrusses: Die Argumente sind verbraucht, die Rituale wiederholen sich, die Debatte nähert sich dem Ende. In der Politik wäre 
dies der Zeitpunkt, eine Entscheidung herbeizuführen und abstimmen zu lassen.

Hier liegt bereits ein entscheidender Unterschied zur Rechtschreibdiskussion: die Sache ist entschieden, allerdings nicht legislativ, sondern exekutiv, und dies nicht allein im nationalen Rahmen, sondern durch überstaatliche Einigung, deren Legitimation selber zwar nicht in Zweifel gezogen wird, deren Voraussetzungen aber nun von den Gegnern der Reform befragt werden. Juristisch läßt sich gegen die überstaatlichen Vereinbarungen nicht angehen, wohl aber gegen die nationalen Auswirkungen: Noch nie hat es bei einem kulturpolitischen Thema in der Bundesrepublik eine solch flächendeckende Anrufung von Gerichten gegeben wie in diesem Fall. Wie der Kruzifix-Streit ist in Deutschland auch die Rechtschreibung beim Bundesverfassungsgericht angekommen, und man ist versucht $\mathrm{zu}$ sagen, daß die Deutschen offenbar nicht bereit sind, Entscheidungen hinzunehmen, sondern sie in jedem Fall juristisch klären zu lassen.

Doch bevor man die Deutschen angesichts dieser Tatsache und vor allem wegen der Aufgeregtheit angesichts dieser Thematik tadelt, sollte man bedenken, daß die Debatte in anderen Ländern, in denen die Änderung der Orthographie geplant oder durchgeführt wurde, ähnlich vehement verlaufen ist. Das war in jüngster Zeit in Frankreich so und auch in den Niederlanden und Belgien, jeweils mit einem wichtigen Unterschied: In Frankreich war es eine Diskussion vor einer Entscheidung ${ }^{1}$, in den Niederlanden anläßlich einer gesetzlichen Regelung. ${ }^{2}$ Dies, wie schon gesagt, war im deutschen Sprachgebiet anders. Ob das erstere, die Diskussion vor der Entscheidung hier verschlafen wurde, ist allerdings selber ein Streitpunkt, worauf noch einzugehen sein wird. Die eher ablehnende Haltung der Öffentlichkeit jedenfalls hatte sich in Deutschland vor der gegenwärtigen Reformdiskussion schon bemerkbar gemacht, gut dokumentiert von Hermann Zabel (Zabel 1989). Andererseits hatte es lange davor und immer wieder Ansätze zu einer Reform der deutschen Orthographie gegeben, von radikalen Vorschlägen wie der Abschaffung der Substantivgroßschreibung bis zur Beseitigung von kleineren Inkonsequenzen der

I Die außerordentlich kontrovers geführte Debatte führte dazu, daß das der Académie Française vom 'Hohen Rat für die französische Sprache' vorgelegte und von ihr zunächst gebilligte Reformkonzept im Januar 1991 wieder zurückgezogen wurde. (Vgl. Hans-Hagen Bremer: 'Der Accent circonflexe und die Sprache Molières. Wie 1991 die Reform der französischen Rechtschreibung nach heftigen Wortgefechten den Tod fand.', FR 8.8.97).

2 Das Gesetz, mit dem vor allem Doppelformen bei Fremdwörtern bereinigt wurden und eine offizielle Wörterliste von etwa 100.000 Wörtern verabschiedet wurde, wurde 1995 von einem niederländisch-flämischen Ministerrat beschlossen. (Vgl. Christine Kasper: 'Die Rechtschreibreform - ein konsekwentes Gräuel', Die Furche 14.11.1996). 
Regelung von 1901. Hier ist nicht der Ort, darauf einzugehen. ${ }^{3}$ Doch muß uns zumindest der anzunehmende rechtliche Status der Orthographie noch beschäftigen, um am Schluß die Debatte einordnen zu können. In jedem Fall ist der Status der Orthographie nicht deutlich. Dies gilt bereits für die linguistische Bewertung bei den Sprechern. Sehr häufig wird die Rechtschreibung einfach mit der Sprache selber gleichgesetzt, und Änderungen der Schreibung werden vielfach als direkte Eingriffe in die deutsche Sprache empfunden. Richtig an dieser Auffassung ist, daß es zwischen Schreibung und Aussprache vielfältige Interdependenzen gibt. Andererseits ist eine orthographische Regelung auch nicht einer administrativen Verordnung wie der für die Verkehrszeichen vergleichbar, ganz gleich, ob die Rechtschreibung, wie im deutschen Sprachraum bis ins 19. Jahrhundert, durch ein Wechselspiel von Vorschlägen grammatischer Autoritäten, Praktikabilitätsgesichtspunkten von Verlegern und Schreibvorschriften der Schulen zustandegekommen ist oder durch eine vertragliche Vereinbarung, die eine exekutive Umsetzung erfahren hat, wie nach der Rechtschreibkonferenz von 1901. Der Staat hat weder ein Sprach- noch ein generelles Schreibmonopol. Aber er hat eine durchschlagende normsetzende Gewalt auch in diesem Bereich. Dies hat sich etwa in der Abschaffung der deutschen Schreibschrift in den Schulen in den vierziger Jahren gezeigt. Seit der Zeit ist diese Schriftart tot. Man darf unterstellen, daß derartige Bedingungen, zumindest vage, bewußt sind, wenn es zu Verabredungen über die Änderung von orthographischen Regelungen kommt. Die Legitimierung der Reform, ihre materiellen Inhalte und ihre Umsetzung werden befragt, das läßt sich nicht vermeiden bei einem Bereich, der alle angeht. Und es kann auch weder erwartet werden, daß sich nur Fachleute zum Thema äußern, noch daß die Auseinandersetzung in quasi wissenschaftlichen Bahnen verläuft. Wir werden sogleich sehen, daß dies überhaupt nicht der Fall ist, im Gegenteil, es bricht ein Streit auf, der vor nichts zurückscheut, bis hin zum Korruptionsvorwurf, so daß es naheliegt, hier eine sich verselbständigende oder gar politisch gesteuerte Diskussion zu vermuten. Und angesichts jedenfalls in der Bundesrepublik anstehender größerer Reformthemen, von der Gesundheits- über die Steuer- bis zur Rentenreform und dem Verdruß darüber, daß diese Reformdebatten auf der Stelle treten, wird vielfach vermutet, die Rechtschreibreform sei ein Gebiet, bei dem einmal eine Reform - vielleicht stellvertretend für die anderen - durchkommen könne - oder aber gerade zu verhindern sei.

Ob dies tatsächlich der Fall ist oder ob nicht auch andere Gesichtspunk-

3 Vgl. zu den Vorschlägen für eine Neuregelung der deutschen Orthographie Nerius et al. (1989), Internationaler Arbeitskreis für Orthographie (Hg.) (1992), Munske (1997) und Augst/Blüml/Nerius/Sitta (Hg.) (1997), zu den jüngsten Reformdiskussionen Nerius (1997) und Scheuringer (1997). 
te zu berücksichtigen sind, um die Debatte gerecht zu bewerten, möchte ich im folgenden darstellen. Es geht mir nicht um die Inhalte der Reform. ${ }^{4}$ Sie spielen, wie ich schon angedeutet habe, in der öffentlichen Diskussion auch eine untergeordnete Rolle. Sie sind, wie großenteils erst im Zuge der Reformdiskussion deutlich geworden ist, viel schwerer zu beurteilen, als es im öffentlichen Bewußtsein und auch bei den Fachleuten verankert ist: ${ }^{5}$ Die Rechtschreibregeln des Deutschen gehorchen keinem einheitlichen Prinzip. Das behauptet auch niemand. Nur wird das phonetisch-phonologische Grundprinzip, das Korrespondenzprinzip, nicht einfach durch das morphematische und andere Prinzipien ergänzt. Sondern das morphematische oder Stammprinzip ist auf dem Wege, der deutschen Orthographie eine andere Fundierung zu geben. Wir nähern uns zwar wohl nicht einer Morphemschrift, aber die blockweise Erfassung von Einheiten beim Lesen, zu der das Stammprinzip vor allem mit der Umlautskennzeichnung und der Nichtkennzeichnung der Auslautverhärtung beiträgt, wird durch grammatische Prinzipien unterstützt, insbesondere durch die Großschreibung der Substantive. Zudem ist die synchron vor allem unter didaktischem Blickwinkel schwierige Uneindeutigkeit mancher Regelbereiche, etwa der Dehnungsbezeichnung ein kompliziertes Geflecht, in das sich kaum eingreifen läßt, ohne daß sich Weiterungen ergeben, die gar nicht beabsichtigt sind.

Was die orthographischen Regeln des Deutschen im einzelnen betrifft, so sind sie als historisch gewordene funktional - sonst hätten sie sich nicht durchgesetzt. Sie haben im Zuge der Entwicklung aber sehr viel Redundanz erzeugt. Die Regeln sind nicht eineindeutig, und es läßt sich zu fast jeder eine Alternativregel angeben, man denke auch hier nur wieder an die Kennzeichnung der Vokallänge. Jede administrative Änderung trägt das Risiko, daß ihr die möglichen Alternativen vorgehalten werden. Die Reform hat deswegen auch kein rigoroses Eingreifen beabsichtigt.

Das Rechtschreibthema beschäftigt die Öffentlichkeit über so gut wie alle Kanäle: von den Printmedien über Hörfunk und Fernsehen bis zum Internet. Ich beschränke mich im folgenden auf die Auswertung von $\mathrm{Be}-$ richten, Interviews, Leserbriefen und Kommentaren aus 34 Zeitungen und Zeitschriften. ${ }^{6}$ Die Sammlung ist insgesamt zwar nicht repräsentativ im

4 Diese sind in einer kaum zu übersehenden Zahl von Publikationen dargestellt. Verwiesen sei hier auf: Internationaler Arbeitskreis fuir Orthographie (Hg.) (1992), auf die Zusammenfassung im Sonderheft des Sprachreport (1996), auf die Kommentierungen in Augst/Blüml/Nerius/Sitta ( $\mathrm{Hg}$.) (1997) und in Eroms/Munske (Hg.) (1997), sowie auf die konsequent neue Vorschläge entwikkelnde Darstellung von Zemb (1997).

5 Dazu u. a. Maas (1992), Eisenberg (1995), Munske (1997), Zemb (1997) und Eroms (1997).

6 Bei der Ermittlung und Sichtung des Materials haben mir Katrin Flexeder und 
statistischen Sinne, aber durch ihre relative Vollständigkeit und ihren Umfang aussagekräftig. Insgesamt wurden 1038 Berichte, 86 Interviews, 824 Leserbriefe und 420 Kommentare herangezogen. Die Berichte und die Interviews wurden nur auf ihre Tendenz hin durchgesehen. Die Leserbriefe und Kommentare wurden zusätzlich darauf hin untersucht, welches Argument für die Entscheidung, der Reform zuzustimmen oder sie abzulehnen, ausschlaggebend war. Überraschend war es, daß sowohl die Tendenz, also Zustimmung oder Ablehnung, fast stets ganz offensichtlich war und andererseits ebenfalls der Hauptgrund für die zustimmende oder ablehnende Äußerung fast immer deutlich zu Tage trat.

\section{Die Berichterstattung in den Printmedien}

Über die Rechtschreibreform wird in der deutschsprachigen Presse kontinuierlich berichtet. Abgesehen von Kommentaren und Interviews, auf die noch einzugehen sein wird, befassen sich in den hier untersuchten Zeitungen und Zeitschriften 1038 Artikel mit der Rechtschreibreform. Das Spektrum reicht von kleinen Meldungen bis zu mehrseitigen informativen Darstellungen. Durchweg wird objektiv, sachlich und neutral berichtet. Die Bevölkerung hat dadurch die Gelegenheit gehabt, sich zutreffend über die Inhalte der Reform zu informieren.

Dies ist aber nur die eine Seite. Denn es gibt bei den Berichten auch klare bewertende Signale. Sie sind in den Überschriften zu finden. Ob sie, bezogen auf den Inhalt, positiv oder negativ gemeint sind, ist nicht von vornherein klar; es können auch allgemeine Aufhänger sein, denn die Rechtschreibreform stellt, journalistisch gesehen, eine hervorragende Thematik dar: Sie betrifft die gesamte Bevölkerung, sie wird kontrovers beurteilt, tendiert damit, wie die Berichte aus dem politischen Bereich, zur Polarisierung, und sie wird im späteren Verlauf unter der Perspektive legislative versus exekutive Befassung diskutiert. Sie ist aber längst nicht von der Brisanz echt politischer Themen, dazu wird die Thematik im Zuge der Debatte erst allmählich stilisiert.

Die Überschriften tragen in besonderem Maße dazu bei, daß die an sich trockene und akademische Thematik anschaulich aufbereitet und mit sonstigen öffentlichen Themen verbunden wird. Sie sind es vielfach, die zur Beschäftigung mit der Rechtschreibthematik reizen. Das ist sicher ihr primärer Zweck, also ein klassisch journalistischer. Aber nebenbei wird dadurch erreicht, daß das Engagement in Bezug auf die Rechtschreibreform zunimmt, und zwar in der angedeuteten Richtung, nämlich polarisierend.

Larissa Vogl, Passau geholfen, denen ich hiermit sehr herzlich danke. Die Grafiken hat Katrin Flexeder angefertigt. Benutzt wurde u. a. das Material des Innsbrucker Zeitungsarchivs. Norbert Richard Wolf, Würzburg, bin ich für klärende Diskussionen sehr zu Dank verpflichtet. 
Die Masse der Überschriften ist neutral. Solche Überschriften sind etwa „Rechtschreibreform ab 1997“ (OÖN 5.7.95)

„Rechtschreibreform zwischen Hoffen und Bangen“" (Die Welt 21.9.95) „Rechtschreibreform verschoben“ (Tsp 28.10.95)

„Jetzt heißt es umlernen auf modifizierte Großschreibung - Was uns die neu beschlossene Rechtschreibreform an neuen Regeln bringen wird" (SN 5.6.96)

„Bundestag debattiert über Rechtschreibreform“ (StZ 15.4.97)

Auch der folgende Typ, der vieles anspricht, kann dennoch als neutral bezeichnet werden:

„Der verkannte Filosof oder: Ein wahrer Alptraum - Die geplante Rechtschreibreform führte zu einer Sprachstörung zwischen Fachwelt, Politik und der Öffentlichkeit." (FR 27.9.95)

Eine, wenn auch unterschiedlich starke oder deutliche Zustimmung signalisieren etwa folgende Überschriftstypen:

„Neue Diskussion, Zeitplan gefährdet?“ (SN 13.9.95)

„Stickel optimistisch“ (FR 22.9.95)

„Sprache im Fluß - Nach jahrelangen Verhandlungen um die Reform der deutschen Rechtschreibung werden die Schulanfänger schon im Herbst nach den neuen Regeln richtig schreiben lernen." (FF 4.5.96)

„Auch mit der Gämse zufrieden - In der mehrsprachigen Schweiz ist eine Änderung der Schreibweise kein Sakrileg“ (FAZ 10.1.97)

„Reformverweigerung von Österreichs Autoren“ (OÖN 13.2.97)

„Neue Rechtschreibung in der Praxis: Jetzt bessern die Schüler die Lehrer aus. - Die Rechtschreibreform wird in einigen Schulen bereits angewendet: Dort stiftet sie nur mäßige Verwirrung." (Die Presse 17.3.97)

Die Zustimmung kann, wie man sieht, direkt oder indirekt signalisiert sein, etwa indem ein Ausdruck mit negativen Konnotationen gewählt wird wie „Reformverweigerung“.

Distanz oder Ablehnung lassen die folgenden vermuten:

„Rechtschreibreform irritiert auch Verlage“ (StZ 13.9.95)

„'Rechtschreibreform gescheitert'“ (Dol 21.9.95)

„Vor der Entscheidung der Ministerpräsidenten: Letzte Warnung vor dem 'Kauboi' - Politiker äußern Bedenken.“ (SZ 26.10.95) 
„Rechtschreibreform kostet einige hundert Millionen.“ (Tsp 1.7.96)

„Daß oder dass? Sprachreform nicht am Parlament vorbeimogeln - In Deutschland Verfassungsbeschwerde gegen geplante neue Rechtschreibung." (OÖN 13.7.96)

„Wer ist der Beste im ganzen Land? Duden contra Bertelsmann: Der Kampf im Rechtschreibdschungel." (SZ 31.8.96)

„Gegen die Rechtschreibreform formiert sich Widerstand - Autoren und Verleger kritisieren die neuen Regelungen - Kritik auch am 'Duden'.“ (SN 7.10.96)

„'Orthographischer Terrorismus': Die deutschen Schriftsteller und Intellektuellen sind jäh erwacht. Drei Monate nachdem die Rechtschreibreform definitiv beschlossen wurde, gehen sie gegen die Neuregelung auf die Barrikaden. Der späte Protest hat überraschend großen Zulauf. Frankfurter Erklärung in Auszügen ..." (taz 8.10.96)

„Rechtschreibreform: Protest prallt an Gehrer ab" (Der Standard 28.10.96)

„Eduscho, zart besaitet - Babylonische Beratung: Neun neue Wörterbücher im Test." (FAZ 15.3.97)

„Reformstopp erwartet“ (Dol 5.6.97)

„Gütersloh und Mannheim liegen bei Babylon: Die Rechtschreibreform hat bislang nur Verwirrung gestiftet, und das Durcheinander wird von Tag zu Tag größer." (FAZ 19.6.97)

„Desaster für Schulen - Urteil bedeutet voraussichtlich Ende der Rechtschreibreform." (Erlanger Nachrichten 30.7.97)

„Möglicherweise folgenreiches Gerichtsurteil in Hessen: Die Rechtschreibreform bereits gescheitert?" (NZZ 30.7.97)

„Die Administratoren der Rechtschreibreform rücken zusammen: 'Jetzt nur keine Panikreaktion' - Durchhalteparolen aus Österreich, Deutschland und der Schweiz - Aus Äußerungen von Bundeskanzler Klima läßt sich schließen, daß man an höchster Stelle auch eine babylonische Schreibverwirrung in Kauf nehmen würde." (Der Standard 31.7.97)

„'Die wollen durch die Wand”“ (Der Spiegel 4.8.97) 
„'In die Falle gelaufen'“ (Der Spiegel 4.8.97)

„Rechtschreibreform: Für die Kultusminister der Länder wird die Luft immer dünner - Wiesbaden, Hannover und Gelsenkirchen - Immer mehr Gerichte lehnen das geplante Regelwerk ab.“ (Allgemeine Staatszeitung 16.8.97)

Insgesamt sind die stellungnehmenden Überschriften aber selten. Zum ganz überwiegenden Teil lassen die Überschriften die Quelle für Pro oder Kontra erkennen, wenn sie nicht gänzlich neutral sind. Oder aber sie sind, wie etwa die beiden Überschriften aus dem Spiegel vom 4.8.97, als Zitat kenntlich gemacht. Der folgende Fall ist daher die absolute Ausnahme. Über einen neutralen ausführlichen Bericht von Hans Wellmann setzt die Zeitung (Gegenwart 1.10.96) die auch sonst deplazierte Überschrift: „Die 'neue' Rechtschreibung. Ein vollständiger, erschöpfender und abschließend wertender Kommentar zur sinnlosesten Reform seit Martin Luther.", kommentiert das allerdings in einer Fußnote mit den Worten „Titel und Untertitel des Aufsatzes stammen nicht vom Autor; sie sollen vielmehr eine gewisse grundsätzliche Skepsis der Red. dieser und allen Rechtschreibreformen gegenüber zum Ausdruck bringen."

\section{Die Meinungsäußerungen in den Printmedien}

Wenden wir uns nun den Meinungsäußerungen in der Presse, den Leserbriefen und den Kommentaren zu. Die vorhin angesprochene Polarisierung tritt hier nun ganz offen zutage. Hier zunächst die Zahlenverhältnisse:

Leserbriefe zur Rechtschreibreform

(824 Leserbriefe von Januar 1996 bis Dezember 1997)

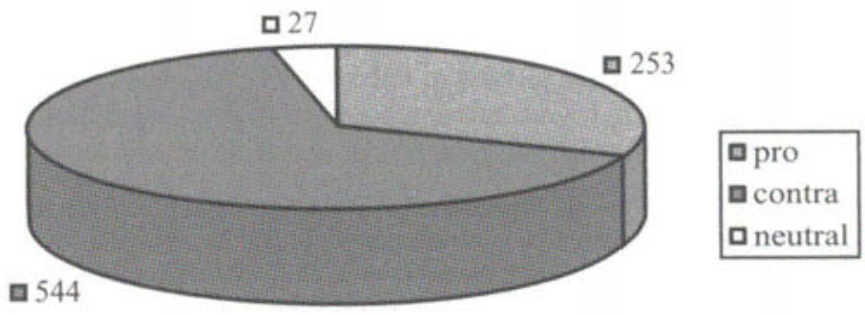




\section{Kommentare zur Rechtschreibreform}

(420 Kommentare von Januar 1996

bis Dezember 1997)

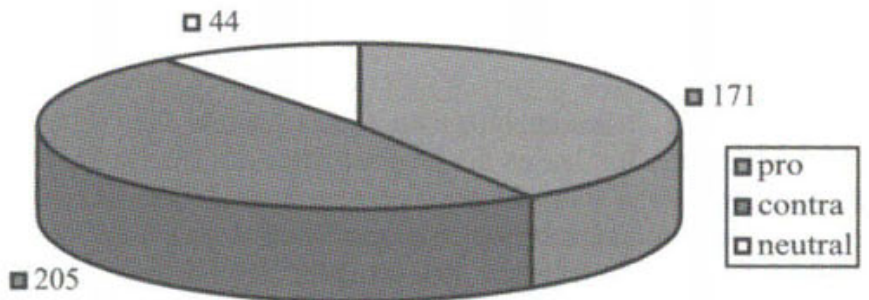

In Prozentzahlen ausgedrückt:

824 Leserbriefe zur Rechtschreibreform (Januar 1996 bis Dezember 1997):

Kontra: $\quad 66,0 \%$

Pro: $\quad 30,7 \%$

Neutral: $\quad 3,3 \%$

377 Pressekommentare zur Rechtschreibreform (Januar 1996 bis Dezember 1997):

Kontra: $\quad 48,8 \%$

Pro: $\quad 40,7 \%$

Neutral: $\quad 10,5 \%$

Aufschlußreich sind die folgenden Vergleichszahlen zum Prozentsatz der Ablehnung oder Zustimmung zur Reform:

Die Umfrage des Allensbacher Instituts Herbst 1996 ergab für die Bundesrepublik Deutschland: Von je 100 Befragten sind 12 für eine Rechtschreibreform, 75 dagegen, 13 sind unentschieden. ${ }^{7}$

Schließlich noch die Verhältnisse bei den Interviews:

Kontra: $\quad 60,3 \%$

Pro: $\quad 36,3 \%$

Neutral: $\quad 3,4 \%$.

7 Präsentation und Analyse der Ergebnisse bei Piel (1997). Zeitgleich mit der Umfrage in Deutschland ergab eine Umfrage des Meinungsforschungsinstituts Fessel + GfK bei 500 Personen in Österreich sehr ähnliche Ergebnisse: $75 \%$ fanden die Rechtschreibreform „schlecht“, 17\% „gut“ (Die Presse 28.10.96). 
Interviews zur Rechtschreibreform

(108 Interviews von Januar 1996

bis Dezember 1997)

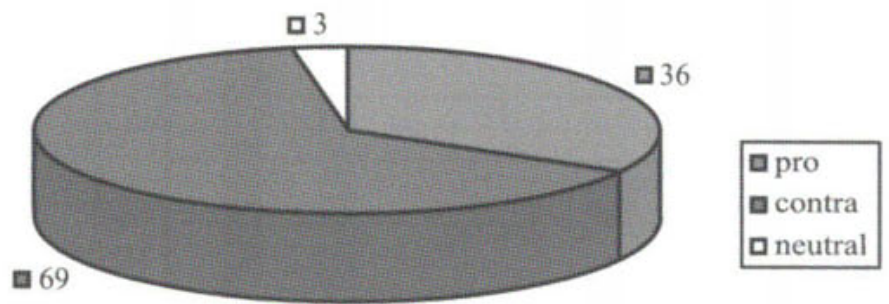

Für die Bewertungen darf man vermuten: Es besteht offenbar ein Zusammenhang zwischen intensiverer Auseinandersetzung und Zustimmungsbereitschaft. Doch gilt dies nicht einfach schlechthin. Die Auffassung der Rechtschreibfachleute ist hier nicht erfaßt, sondern die erfragte Meinung im Verhältnis zu der sich selbst artikulierenden, vor allem aber das Verhältnis von artikulierter Meinung in den Leserbriefen zu der in den Kommentaren. Dies spiegelt sich nicht zuletzt im Prozentsatz der neutralen Stellungnahmen. Bei den Kommentaren ist ein deutlich höherer Anteil die Argumente abwägender Texte festzustellen als bei den Leserbriefen. Dies ist verständlich, aber durchaus nicht selbstverständlich, die Heftigkeit der Argumentation bei den Stellung beziehenden Texten ist bei Leserbriefen und Kommentaren durchaus ähnlich.

Was die zeitliche Verteilung betrifft, so läßt sich erkennen, daß die Zahl der Leserbriefe und Kommentare nach der 'Frankfurter Erklärung' (6.10.96) und nach den ersten Gerichtsurteilen (ab Juli 1997) stark ansteigt. (Vgl. Graphiken auf S. 204 f.)

Leserbriefe und Kommentare sind nicht nur nach der Tendenz ausgewertet, sondern es ist versucht worden, ihr jeweiliges Hauptargument für die Entscheidung zu ermitteln. Die Argumente wurden sodann in acht Kategorien zusammengefaßt. Es sind Paradebeispiele von „Argumentationstopoi“ im Sinne von Jung/Wengeler (1998). (Vgl. Tabellen auf S. 205f.)

Die Rubriken tragen in den Tabellen prägnante Kurzfassungen für die geäußerten Ansichten. Sie wurden in jeweils acht Kategorien zusammengefaßt und diese nach ihrem prozentualen Anteil geordnet. Dabei ergaben sich sowohl für die ablehnenden als auch für die zustimmenden Äußerungen im Vergleich von Leserbriefen und Pressekommentaren erstaunliche Übereinstimmungen, allerdings ist die Diktion in Leserbriefen und in Kommentaren unterschiedlich. Die gröberen Ausdrucksweisen entstammen überwiegend, aber nicht ausschließlich den Leserbriefen. 


\section{Leserbriefe zur Rechtschreibreform}

(1996/97)

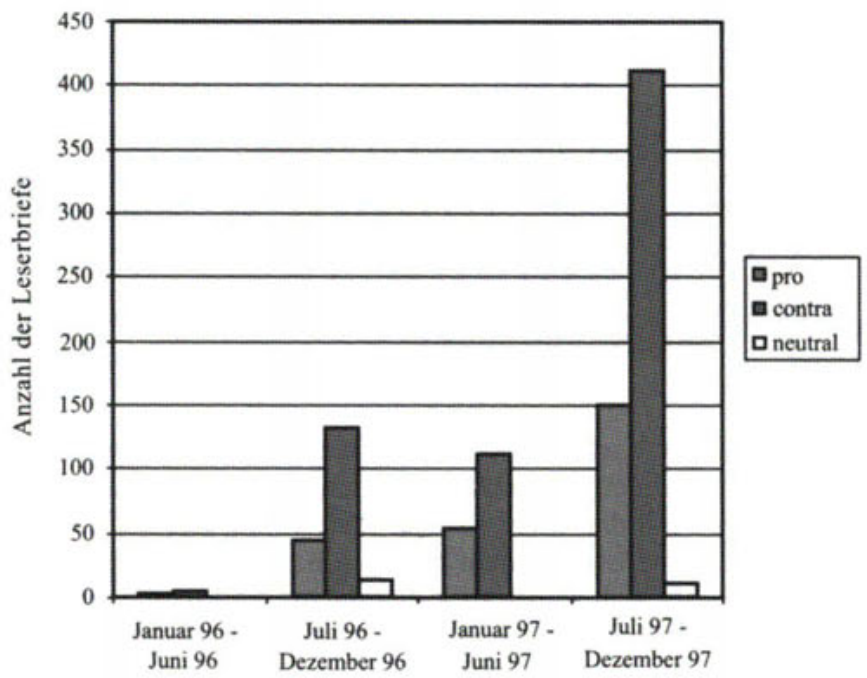

Kommentare zur Rechtschreibreform

(1996/97)

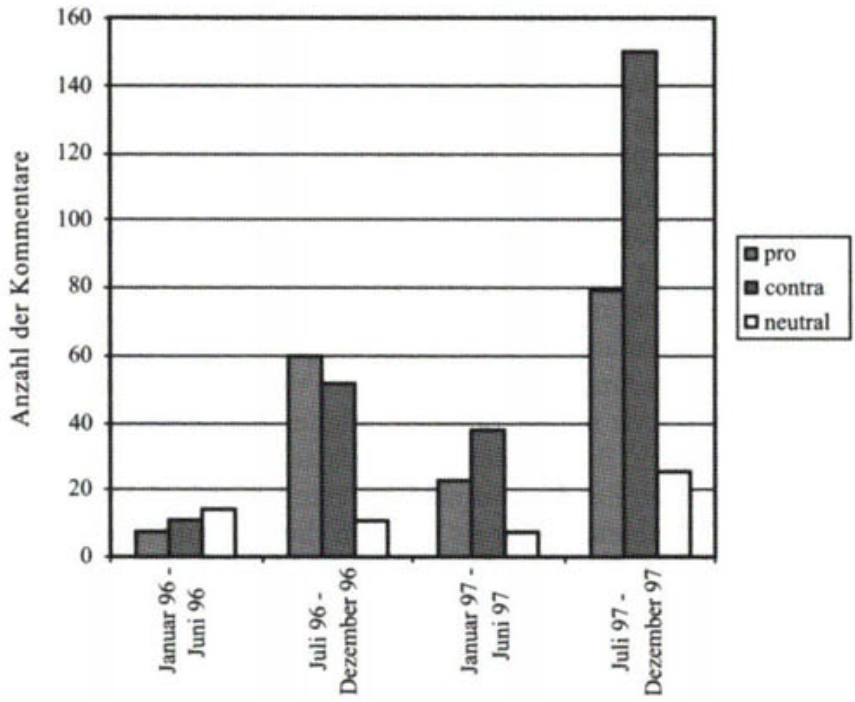


Interviews zur Rechtschreibreform

(1996/97)

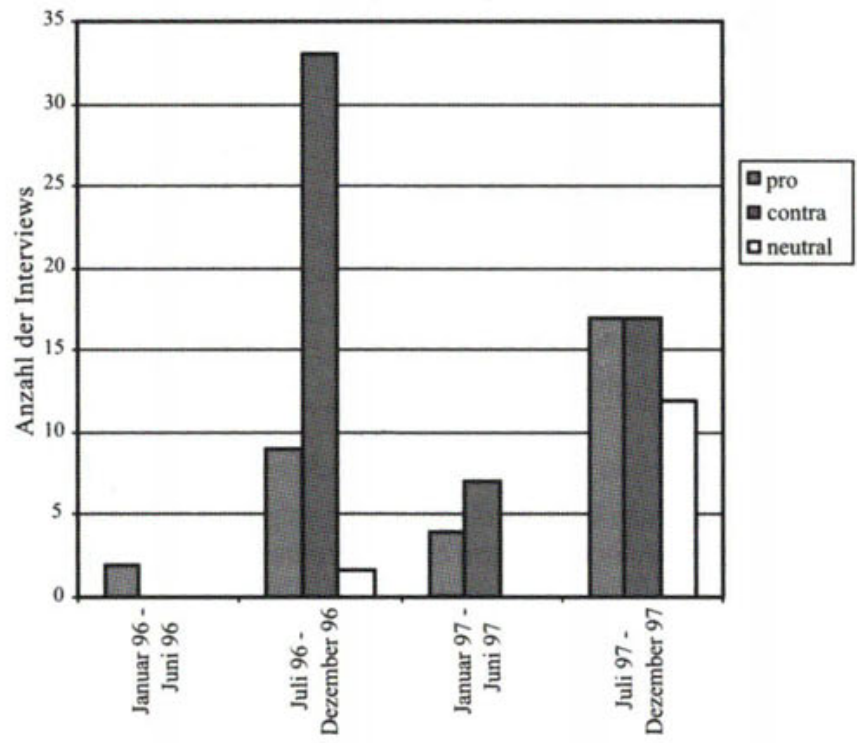

Kontra

1. Die Reform ist mißglückt

Leserbriefe Kommentare

2.

... ist obrigkeitlich

3. $\quad \ldots$ ist ein ,Mäuslein“

$30,0 \%$

$38,1 \%$

4. $\quad \ldots$ verletzt die Tradition

$20,6 \%$

$30,2 \%$

5. Einzelheiten sind mißglückt

$9,0 \%$

$6,3 \%$

6. Die Reformer sind arrogant

$13,6 \%$

$5,9 \%$

7. Begleitumstände sind zu kritisieren

$7,9 \%$

$6,3 \%$

8. Die Reform ist Aufhänger für anderes

$7,2 \%$

$6,8 \%$

$5,7 \%$

$2,4 \%$

$6,1 \%$

$3,9 \%$ 


\begin{tabular}{|lccc|}
\hline \multicolumn{3}{|c|}{ Pro } \\
\hline & Leserbriefe & Kommentare \\
1. Die Reform ist geglückt & $35,2 \%$ & $36,3 \%$ \\
2. & $\ldots$ ist legitim & $4,3 \%$ & $11,1 \%$ \\
3. & ... beseitigt Monopole & $1,2 \%$ & $3,5 \%$ \\
4. & $\ldots$ geht mit der Zeit & $15,4 \%$ & $14,0 \%$ \\
5. Einzelheiten sind mißglückt & $2,0 \%$ & $2,3 \%$ \\
6. Die Reformgegner sind hysterisch & $34,8 \%$ & $27,5 \%$ \\
7. Begleitumstände sind zu kritisieren & $2,0 \%$ & $0,6 \%$ \\
8. Die Reform ist Aufhänger für anderes & $5,2 \%$ & $4,7 \%$ \\
\hline
\end{tabular}

Die acht Bewertungskategorien umfassen folgende Argumente, die im Auswertungsteil noch mit weiteren charakteristischen Formulierungen belegt werden:

\section{A. Ablehnungen}

1. Die Reform ist als Ganzes mißglückt, sie führt zur Verwirrung, ins Chaos, Simpeldeutsch, gehört auf den Müll.

2. Sie ist von der Obrigkeit, der Bürokratie, oktroyiert, sie ist rechtlich ungeklärt, ihre Umsetzung Ausfluß vorauseilenden Gehorsams.

3. Ein Berg kreißt, ein Mäuslein wird geboren. Die Reform ist überflüssig wie ein Kropf. Sie ist ein Mückenschiss.

4. Die Reform verletzt die Tradition, das historische Erbe.

5. Die Reform ist in Einzelheiten mißglückt.

6. Die Reformer sind arrogant, anmaßend, inkompetent, sie lügen.

7. Es wird an Begleitumständen Kritik geübt.

8. Sie wird als Aufhänger genommen, um anderes zu kritisieren.

B. Befürwortungen

1. Die Reform ist als Ganzes gelungen, sie erbringt eine Vereinfachung.

2. Die Reform ist rechtlich in Ordnung.

3. Das Dudenmonopol fällt.

4. Die Reform reagiert auf die Entwicklung der Sprache.

5. Die Reform ist in Einzelheiten geglückt.

6. Die Gegner sind hysterisch, sie blockieren, sie sind Populisten, ihr Protest ist übertrieben oder kommt zu spät.

7. Es werden Begleitumstände positiv hervorgehoben.

8. Sie wird als Aufhänger genommen, um anderes zu kritisieren. 
Diese jeweils acht Kategorien, die sich beim Ordnen der insgesamt über eintausendzweihundert Texte als Sammelkategorien aus dem Material ergeben haben, lassen unter anderem folgendes gut erkennen: Die positiven und die negativen Bewertungskategorien entsprechen einander weitgehend. Es sind einerseits sachbezogene, darunter wieder globale und partielle. Insbesondere ist es aufschlußreich zu sehen, daß die Frage der Legitimierung der Reform und der Bezug auf die geschichtliche Entwicklung gleichermaßen gesehen und artikuliert wird - natürlich mit umgekehrten Vorzeichen. Andererseits finden sich auch analoge Kategorien bei der personenbezogenen Argumentation und bei grundsätzlichen Stellungnahmen zur deutschen Sprache.

\subsection{Die Leserbriefe}

Als erstes sollen die Leserbriefe genauer betrachtet werden. Sie sind in ihrer Argumentation und in ihrer Diktion sehr unterschiedlich. Es äußern sich sehr häufig an der Reform Beteiligte oder aber profilierte Gegner. Doch es sind auch Schreiber dabei, die offensichtlich normalerweise nicht zur Feder greifen. Immer ist zu bedenken: Wer sich äußert, outet sich, er oder sie bezieht Stellung. Wir werden sehen, daß dies bis zum Ablegen eines Bekenntnisses geht. Schon dies relativiert die sachlichen Bezüge, die man eigentlich erwarten würde. Bis auf ganz wenige Briefe, die dann auch immer um die gleiche Problematik kreisen (die neue $s$-Regelung, die Kommaregeln, die Fremdwörter und die vereinzelten etymologischen Neuregelungen), spielen Sachfragen überhaupt eine untergeordnete Rolle. Es geht den Schreibern überwiegend um Grundsätzliches.

Unter den noch eher sachnahen Argumentationslinien der Reformgegner sind solche zu nennen, die die Ergebnisse der Reform als mager ansehen im Verhältnis zum Aufwand, der dazu geführt hat. In der Tabelle sind diese im Argumenttopos 3 als „Mäuslein“" und „Kropf“ markiert. Sie lassen sich deswegen zusammenfassen, weil sie auch vielfach in den Leserbriefen zusammen so benannt werden: „Der Berg kreißt und gebiert ein Mäuslein“. Und das Wort des Bundespräsidenten, die Reform sei überflüssig wie ein Kropf, findet sich schon vorher: Man beachte beim ersten Zitat, daß und wie der Schreiber argumentiert:

„Aber ein anderer Schluß ist erlaubt und drängt sich geradezu auf. Wenn die Reform kaum bemerkbar ist, ist sie dann nicht - überflüssig wie ein Kropf? Wäre es nicht aberwitzig, wegen dieses winzigen und lächerlichen Reformmäusleins, das sprachwissenschaftliche Berge nach vieljähriger Schwangerschaft schließlich geboren haben, Abermillionen Mark auszugeben für neue Schulbücher, neue Behördenformulare, neue Lexika usw.?" (SZ 6.10.95)

Häufig wird vom „Reförmchen“ (z.B. Die Zeit 13.10.96) gesprochen, 
übrigens auch von den Anhängern. Die Reform sei ,von weltfremden Theoretikern ausgeheckt" (DER SPIEGEL 28.10.96) oder sie sei ,ganz offensichtlich nur zur Profilierung einiger Eierköpfe geeignet, die auch mal als 'Reformer' in die Geschichte eingehen wollen." (PNP 31.10.95)

Eine beeindruckende Bildermischung findet sich in einem Leserbrief in der Mittelbayerischen Zeitung vom 2./3.8.97: „Diese Reform ist eine Fehlgeburt. Niemand von den Verantwortlichen will jetzt zugeben, einen Bock (des Jahrhunderts) mitgeschossen zu haben. Die Zeche zahlt (wieder mal) der Bürger - und die (Schulbuch-)Verlage reiben sich die Hände." Diese unkoordinierte Metaphernfülle ist sicher in erster Linie Ausdruck der Betroffenheit des Schreibers.

Das „Kropf"-Argument findet sich in mannigfachen Variationen, gerne als Schluß einer Argumentenkette, z. B. in der Frankfurter Allgemeinen Zeitung vom 20.8.97: „Den einen bleibt die Peinlichkeit erspart, nach gewaltigem Kreißen ein Mäuslein zu gebären. Die andern werden vor einem Kropf bewahrt, den sie für überflüssig halten."

Die Gegner sorgen sich auch in diesem Zusammenhang ums politische Gemeinwohl: „Wenn aber ein Vorhaben nichts bringt außer Verwirrung und Kosten, sollte man es bleibenlassen. Auch im Interesse der Glaubwürdigkeit. Denn wie könnten die Bürger den Politikern bei notwendigen, aber schwer durchschaubaren Reformen wie zum Beispiel der Rentenreform noch vertrauen, wenn ein für alle erkennbarer Schwachsinn wie die sogenannte Rechtschreibreform durchgeboxt würde?" (SZ 5.5.97). Hier wird der vom Spiegel (14.10.1996) in die Welt gesetzte Ausdruck „Schwachsinn“ weitergetragen.

Mit dem Hinweis, die Reform führe zu einem Bruch mit der sprachlichen Tradition (Argumentengruppe 4), wird von den Gegnern ein rein formal gesehen nicht zu widerlegendes Argument angeführt. Denn jede Änderung setzt zwangsläufig Altes, Herkömmliches außer Kraft. Von den Reformbefürwortern wird dem meist entgegengehalten, daß die Sprache und damit ihre verschriftete Form sich entwickelt habe und der Anpassung bedürfe. Die Reformgegner gehen zumeist ins Grundsätzliche.

„Obwohl erfahrungsgemäß Dummheit kaum heilbar ist, sei den eifrigen Reformern doch gesagt: Es wäre zu bedenken, daß Sprache nicht einfach ein modernisierbares Werkzeug ist, sondern Abbild der Kulturtradition und daß die Auslöschung des Traditionsbewußtseins den Menschen auf die gleiche Ebene mit dem Tier setzt." (SN 8.11.95). Ist hier ein geradezu fundamentalistischer Bezugsrahmen gesetzt, ist es im folgenden Leserbrief immerhin noch ein sozialpolitischer: „Das Streben nach einer sozialen Hängematte für alle in Not Geratenen und Durchhänger kann nicht auf die Sprache übertragen werden, sie kann nicht zu einer Hängematte für Rechtschreibschwache werden. Kinder und Erwachsene, die mit ihrer Muttersprache Schwierigkeiten haben ..., werden sie auch bei einer ver- 
einfachten Rechtschreibung zeigen." (Die Welt 12.12.95). In beiden Fällen werden grundsätzlich politische Anliegen aufgearbeitet.

In anderen Briefen werden Angstszenarien entwickelt: „Die Rechtschreibreform beraubt uns ohne Not eines Teiles unserer kulturgeschichtlichen Vergangenheit. Heute schreiben sie uns vor, wie wir zu schreiben haben. Morgen werden sie uns diktieren, was wir zu schreiben haben. Noch ist Zeit, etwas dagegen zu unternehmen." (DER SPIEGEL 28.10.96). Auf die Furcht vor der anonymen Obrigkeit, die sich hier bemerkbar macht, wird noch einzugehen sein. Andererseits sucht man die Rettung dort, wo sie nach der Meinung anderer nun gerade nicht verankert sein soll: „Konrad Duden hat sich im Grab umgedreht, ich habe es deutlich gehört.“ (SZ 2./3.11.96)

Für viele Kritiker ist das Vereinfachungsargument bei der Reform unerträglich. Es wird auf die Schwierigkeit beim Schreiben etwa des Japanischen, Französischen und Tschechischen verwiesen (z.B. Die Furche, 28.11.96). „Nach dem Krieg sind den Wurzeln unserer Traditionen, unserer Werte schon unübersehbare Wunden geschlagen worden. Soll nun ein weiteres, ganz wichtiges Schlüsselelement geschwächt werden?"“ (Die Welt 26.8.97)

Die Beispiele lassen sich vermehren. Deutlich ist, daß hier diffuse Ängste aufbrechen, Ängste auch, die sich auf den Einführungsmodus der Rechtschreibreform beziehen. In der Graphik ist dies in der Rubrik 'Obrigkeit' erfaßt.

In der Frankfurter Rundschau vom 21.8.96 heißt es in einem Leserbrief: „Der preußische (oder hier doch: Preußische?) Obrigkeitsstaat läßt - nein nicht grüßen, sondern antreten zur Rechtschreibreform. “ Oder: „Die Sprache ist zu wertvoll, um sie dem Kultusminister oder irgendwelchen Kommissionen zu überlassen, die durch solche Reformen ihre Existenzberechtigung nachweisen zu müssen glauben." (SZ 30.10.96)

Hier und in manchen anderen Leserbriefen wird damit auch die Legitimation der Kommission angezweifelt:

„Es ist nachvollziehbar, daß die lediglich aus Linguisten und Pädagogen zusammengewürfelten Mitglieder des Mannheimer Instituts für deutsche Sprache (IDS) und des Internationalen Arbeitskreises für Orthographie, die nun schon seit Jahrzehnten ohne jeden Erfolg an einer - tatsächlich allenfalls marginal sinnvollen - Rechtschreibreform herumgebastelt haben, endlich einmal einen 'Durchbruch' sehen wollten. Nur für den Papierkorb zu werken ist schon frustrierend. Jahrzehntelanger Frust ist aber eine denkbar untaugliche Motivation für ein Reformvorhaben. Und so ist das, was die beiden Gremien im Elfenbeinturm nun mit Hilfe der ländereigenen Kultusbürokratie ganz nach Art der Duodezfürsten auf kaltem Wege durchzudrücken suchen, jedenfalls partiell ein Desaster, aufs Ganze gesehen zumindest völlig überflüssig. ... Schließlich ist das Verfahren, in dem die Rechtschreibreform, die immerhin ein wesentliches, letztlich jeden Staatsbürger betreffendes Kulturgut tangiert, durchgedrückt wurde, 
eines demokratischen Staates zutiefst unwürdig. Es mag schon sein, daß nicht jede Einzelheit eines Reformvorhabens einer Volksabstimmung oder eines Parlamentsbeschlusses bedarf. Aber jedenfalls hätte eine solche Entscheidung über das $\mathrm{Ob}$ der Reform, die die Reformer zweifellos gescheut haben wie der Teufel das Weihwasser, getroffen werden müssen. " (SZ 8.11.96)

In anderen Briefen wird von einer ,ordre de mufti“ gesprochen (Die Welt 4.12.96), von ,undemokratischem Diktat“ (Die Welt 11.8.97) oder davon, $\mathrm{da} \beta$ der Bevölkerung, die nicht gefragt worden sei, der Mund verboten werden solle (ND 5.3.97).

„Wenn dann auch noch Kulturpolitiker als Bedenkenträger ihre eigene Meinung für die zu schützende derer ausgeben, die sie gar nicht gefragt haben und damit die illustren Kreise besagter Fachkompetenzler quadratieren wollen, dann wird's schon gleich zweimal nichts." (SZ 15.5.97). Dieser Schreiber führt noch eine große Zahl weiterer Gegenargumente an und relativiert ungewollt die zu seinem Thema gehörenden dadurch, daß er wie viele andere auch - die Rechtschreibreform zum Anlaß nimmt, über den ,täglich tonnenweise produzierten Sprachschrott“ zu lamentieren.

Äußerungen wie die folgende sind häufig: „Diese bürokratische Maßnahme ist ein Rückfall in den Obrigkeitsstaat und findet darum in der Bevölkerung auch so geringe Akzeptanz." (MZ 2./3.8.97)

Der Unmut gegen die Obrigkeit richtet sich aber nicht nur gegen diese direkt, sondern sucht auch bei den Betroffenen Mitschuld, insbesondere bei Lehrern, bei Verlagen und allen denen, die ,in vorauseilendem Gehorsam" sich zu Handlangern der Obrigkeit gemacht hätten.

Das Wort vom vorauseilenden Gehorsam fällt mehrfach (z.B. Die Woche 24.1.97; ND 5.8.97; FAZ 13.8.97).

In anderer Ausdrucksweise wird etwa der 'Woche', die die neue Rechtschreibung bereits anwendet, vorgeworfen ,das 'weichgespülte SimpelDeutsch' (Zit.) unters Volk zu bringen. Schade, dass ausgerechnet Sie so schnell weich geworden sind." (Die Woche 3.1.97). In der gleichen Zeitung geht ein Schreiber noch stärker ins Grundsätzliche: „Es ist zu spüren, was Sie im Grunde zur Vorwegnahme der neuen Schreibnorm gebracht hat: das Bedürfnis sich zu zeigen, sich abzuzeichnen und avant zu sein. Es ist respektlos, das Demokratische nicht erst einmal zu beobachten und nicht abzuwarten, welcher Erfolg der Widerstandsbewegung in Schleswig-Holstein und in Bayern beschieden sein wird." (Die Woche 17.1.97)

Was die rechtlichen Fragen betrifft, so wird in den Leserbriefen moniert, daß die Parlamente übergangen worden seien (z. B. SZ 22./23.3.97), was selbstverständlich auch polemisch ausgedrückt wird (,das Vorbeimogeln der Reform durch die Kultusminister im Erlaßwege an den Parlamenten vorbei“", Tsp 4.5.97) und nun als Hebel betrachtet wird, die Reform zu stoppen: „Na endlich. Es haben sich Juristen gefunden, die dem Unfug der 
Rechtschreibreform mit juristischen Mitteln hoffentlich ein Ende setzen. Die wirklich nötigen Reformen sind nicht machbar, wie eine vernünftige Rentenreform oder eine wirkliche Steuerreform." (Die Welt 7.6.97). Hier wird interessanterweise das Argument, der Streit um die Rechtschreibreform sei ein Stellvertreterkrieg, den die Befürworter anführen, gegen die Reform gewandt. Die juristische Seite der Problematik wird vielfach in Karikaturen aufgegriffen, wofür zwei Beispiele stehen mögen.

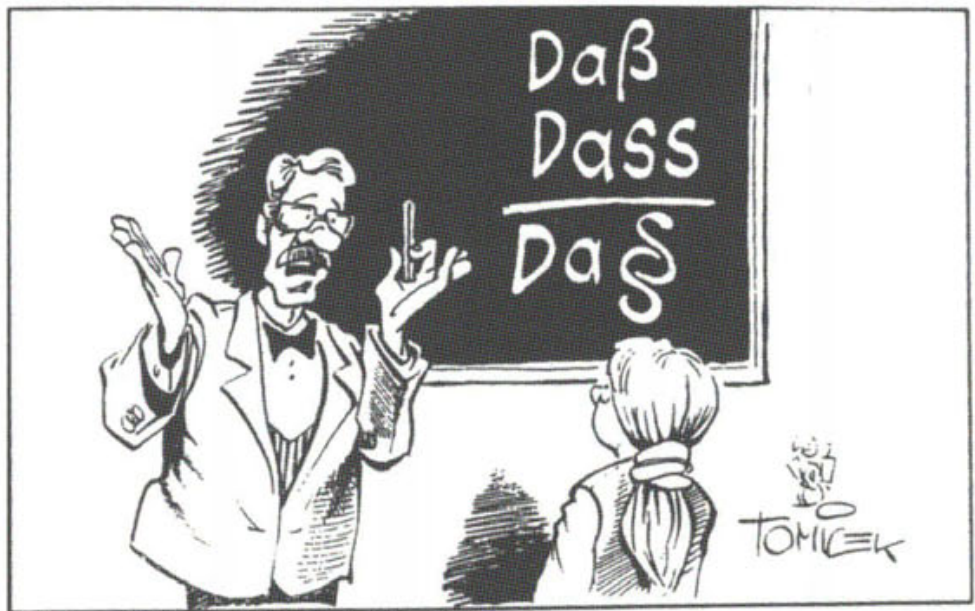

.voriaufige Schreibweise!" ,

Zeichnung: Tomicek

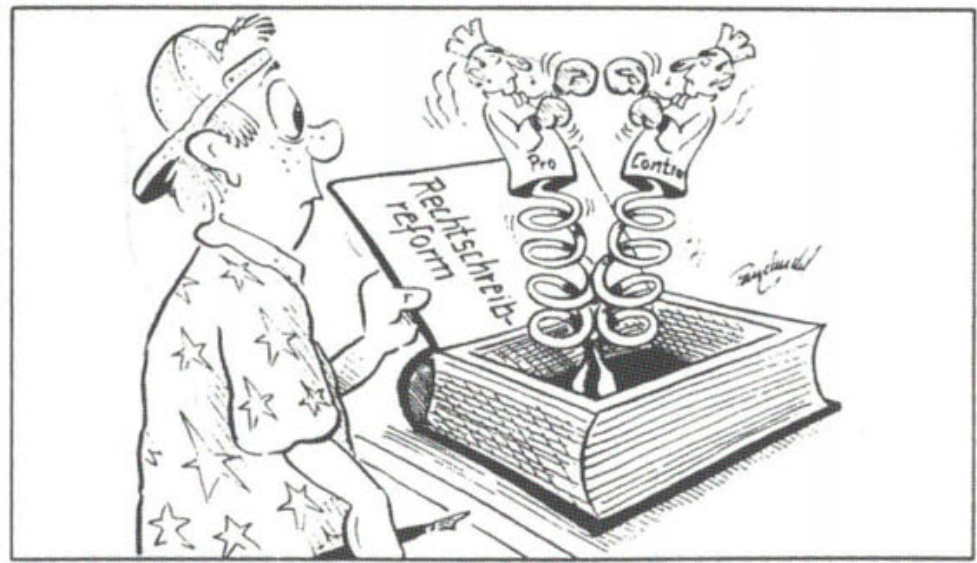


Auch das Rechtsargument wird mit anderen Motiven verknüpft: „Ich bin aber fest davon überzeugt, daß diese Art der Einführung einer sprachlichen Reform auch einer bundesverfassungsgerichtlichen Überprüfung nicht standhalten wird. Denn im Endeffekt soll damit dem deutschen Volk eine Veränderung der Schriftsprache aufgezwungen werden, die, ohne dazu legitimiert zu sein, von ein paar elitären Kreisen ausgeklüngelt wurde.“(FR 11.8.97). Die Kultusminister hätten „frühzeitige Warnungen ... in den Wind geschlagen und statt dessen klammheimlich Fakten geschaffen". (taz 13.8.97)

Besonders aufschlußreich ist es, zu registrieren, daß die Vorbehalte gegenüber der Rechtschreibreform zum Anlaß genommen werden, den Unmut über damit in Zusammenhang Stehendes oder ganz anderes zum Ausdruck zu bringen: So etwa daß die Bewertung von Rechtschreibleistungen nicht mehr den Stellenwert habe wie früher (Die Welt 3.7.96). Es wird auf die Belange der Legastheniker hingewiesen (ND 6.9.96) oder daß mit der Rechtschreibreform der Anglisierung Vorschub geleistet werde (TA 16.9.96). Vor allem aber findet sich immer wieder das folgende Argument:

„Diese Reform ist auch eine Folge unserer Nachlässigkeit im Umgang mit unserer Sprache. Unsere Sprache ist mehr als simpel geworden. Die Verunstaltungen durch die Neuerungen sind nur eine Folge. Hört man den Mann oder die Frau auf der Straße sprechen: Wer von ihnen spricht überhaupt noch ein gutes Deutsch, geschweige einen richtigen Satz? Alles ist nur noch locker, toll, wahnsinnig, super, geil. Damit hören die Sätze im allgemeinen auf. Wer mehr spricht, 'tickt ja nicht richtig' oder ist arrogant oder kommt aus einer anderen Welt. Auch die Schrift unterliegt dem Wandel der Zeit. Wer schreibt heute noch schön? Unsere älteren Herrschaften waren sicherlich nicht alle Schreibtalente. Ich habe aber noch niemanden von den Älteren kennengelernt, 'dem die Schrift auf den Keks ging'. Die Disziplin, die Geduld und die Übung machten es möglich. Aber das Wort Disziplin darf man heute erst gar nicht äußern. Ernste Blicke oder empörte Worte sind die Reaktion." (FAZ 18.10.96)

Ein sehr langer Leserbrief in der Süddeutschen Zeitung vom 26./27.10.96 fuihrt acht Bereiche an, in denen der gegenwärtige Zustand der deutschen Sprache zu kritisieren sei, u. a. der falsche Plural Worte und der falsche Gebrauch des Suffixes -fähig, im Spiegel wird die „Jet-set-Sprache“ beklagt (28.10.96). Selbst die Sprache der Diskutanten wird unter die Lupe genommen, so wenn die Ausdrucksweise „zielt daneben“ eines Leserbriefschreibers durch einen anderen kritisiert wird, es müsse heißen ,trifft daneben". (SZ 2./3.11.96)

Weitere Beispiele erspare ich mir. Hier ist jedenfalls deutlich zu sehen, daß sich die Diskussion verselbständigt hat. Die Rechtschreibreform ist der Anlaß, zu einem Rundumschlag anzusetzen. Dies aber ist nicht auf die Gegner beschränkt, auch die Befürworter tendieren häufig dazu, grundsätzlich zu argumentieren. 
Damit komme ich zu den Reformbefürwortern.

Vielfach werden auch hier durchaus sachliche Argumente herangezogen, auch wenn sie nicht mehr diskursiv entwickelt, sondern eher formelhaft berufen werden. So etwa daß die Reform Vereinfachung und Einheitlichkeit erbringe (DER SPIEGEL 3.7.95, FAZ 18.10.96). Das gleiche gilt für die Behauptung, die Reform sei logisch, maßvoll und angemessen, was vor allem von den leserbriefschreibenden Kommissionsmitgliedern und sonstigen Verantwortlichen häufig vorgebracht wird. Es ist nicht zu verkennen, daß auch hier dogmatisch behauptet wird: „Diese von Fachleuten als gemäßigt und vernünftig eingestufte Rechtschreibreform wird sich durchsetzen, weil sie das Schreiben erleichtert und Fehler verringert." (Die Woche 10.1.97)

Auch die Argumentation, da $\beta$ die Rechtschreibreform konsequent sei, weil sich die Sprache fortentwickle und mithin auch die Schreibung, wird häufig leerformelhaft gegeben: ,, Sicher ist die sogenannte Rechtschreibreform nicht der Weisheit letzter Schluss. Auf alle Fälle ist sie jedoch überfällig: Es ist einfach peinlich, wenn man Kindern erklären muss, dass 'das Essen und Trinken' usw. groß geschrieben werden müssen, 'im großen und ganzen' ... jedoch klein." (Main-Post 2.8.97). Sie gibt Anlaß zu feierlichen Bekenntnissen: „Ich bekenne hiermit: Ja, ich will diese Reform!“ oder: „Die Vereinfachung der Orthographie ist ... vor allem auch ein demokratischer Akt!“ (taz 13.8.97) oder auch: „Die Reform ist für die Zukunft. Die Zukunft sind unsere Kinder. Also laßt doch unsere Kinder in Ruhe und Frieden lernen." (WAZ 19.8.97)

Wie die Gegner, gehen auch die Befürworter der Reform ihre Kontrahenten frontal an. Zwei Vorwürfe finden sich sehr häufig: der Protest sei maßlos, jedenfalls übertrieben und er komme zu spät. „Wirklich niedlich, - da schreibt ein Deutschlehrer aus der bayerischen Provinz einen Protestaufruf gegen die Rechtschreibreform, und unsere Dichterelite stimmt ein mit einem Getöse, als müßte sie in Zukunft mit kyrillischen Buchstaben schreiben." (DER SPIEGEL 28.10.96)

Die Gegner werden als „Handlanger" (FAZ 1.8.97), „Nörgler" (WAZ 5.8.97), die albernen „Rechtschreib-Rumpelstilzchen“ (Main-Post 6.8.97), „konservative Dauerprotestierer" (ND 22.8.97), „Rechtschreibpharisäer“" (Die Presse 23.8.97) bezeichnet oder es wird von „der bekannten Profilierungssucht einiger Personen“ gesprochen (Die Welt 8.8.97), von ,aufgebrachten alten Damen und Herren" (Westf. Rundschau 12.8.97). Das heißt insgesamt auch hier: eine starke Tendenz zur Polemik, zum Verlassen der sachlichen Bahnen.

Dies wird in anderen Bereichen noch erheblich deutlicher: Der Verlokkung, zu einer Generalabrechnung mit den Gegnern der Rechtschreibreform anzusetzen, geben viele Leserbriefschreiber nach. So heißt es in einem Brief an den Münchner Merkur vom 14./15.8.97: 
„Ich schätze Ihre Kommentare ja zumeist sehr. Um so ärgerlicher erscheint es mir, wenn Sie sich - gibt's im Sommerloch keine anderen Themen? - in die zugegeben lange - Schlange der zu spät aufgestandenen Rechtschreibkritiker einreihen. Der Duden ist umgeschrieben, die Schulbücher - mit der Einführung der neuen Lehrplanteile in der Hauptschule - der neuen Rechtschreibung angepaßt. Und jetzt wachen endlich alle auf und wollen auch mitreden und, das scheint in Deutschland Spezialität zu sein, alles wieder so heil und toll machen, wie es früher schon immer war. Typisch ist dann, wenn man vom Kultusminister behauptet, daß 'seine' Rechtschreibreform (vermutlich hat er sich's am Frühstückstisch überlegt! Pfui! Weg mit einem solchen Minister!) ein 'Unfug' ist. Dazu passen die Leserbriefe vom 8. August, in denen einer meint, die Millionen für die Rechtschreibreform (seit den letzten zwei bis drei Jahren) wären bei den Hochwassergeschädigten bestimmt besser angelegt. Ja, hätte er das Hochwasser nur rechtzeitig vorhergesagt! Oder der andere, der meint, die 'abhängigen' Lehrkräfte werden sich auch weiterhin bemühen, sich nicht gegen das Ministerium aufzulehnen. Der hat ja ein tolles Insiderwissen! Übrigens: Meine Fünft- und Sechskläßler sind auf die neuen Regeln ziemlich scharf und freuen sich diebisch, wenn sie in den 'alten' Büchern Fehler entdecken. Aber die Kinder sind halt noch nicht so verknöchert und festgefahren."

Dies ist eine Retourkutsche auf Kommentare und Leserbriefe in der gleichen Zeitung, die den Befürwortern Wichtigtuerei (30. Juli, S. 2) vorgeworfen hatten.

Auch hier hat sich der Streit verselbständigt. Die Rechtschreibreform wird zum Anlaß genommen, vermeintlich typisch deutsche Zustände zu beklagen, insbesondere das „Ewig Gestrige“. Der angefuihrte Leserbrief schließt: „Wobei mir die Abwandlung eines alten Gebetsschlusses in den Sinn kommt: So, wie es war (vor $40 \mathrm{Jahr}$, vor $60 \mathrm{Jahr}$...), so auch jetzt und immerdar und in Ewigkeit. Amen." - Insgesamt eine Trivialversion der Bestimmung Deutschlands als der ,verspäteten Nation“.

Dafür noch einige weitere Beispiele:

„Sommertheater 97. Thema: Wir gehen auf die Barrikaden gegen die Rechtschreibreform. Untertitel: Der deutsche Michel in Aktion. Ein Lehrstück.

Lehre 1: Manchmal ist des deutschen Michel Zipfelmütze eine Schlafmütze. Das Reförmchen wurde lang und breit diskutiert. Der Michel wacht erst auf, als die Entscheidung gefallen ist. Und dann regt er sich auf. Spät, dafür aber mächtig.

Lehre 2: Michel mag keine Veränderungen. Dabei geht es für ihn gleich um das Eingemachte. Die neuen Regeln bedrohen seine Identität, die Vernunft, die Ästhetik, die Kultur, das Abendland. Zur Rettung müssen die Gerichte angerufen werden. Vielleicht tät es auch ein guter Psychiater." (taz 15.8.97, S. 14)

Nach der Frankfurter Erklärung wird gefragt: „Was ist das für ein politischer Stil, erst zu kritisieren, wenn Veränderungen unmöglich geworden sind? Haben die prominenten Autoren, die berufenen Anwender der deutschen Sprache, bisher geschlafen und sind erst aufgewacht, als sie begriffen haben, daß sie selbst etwas dazulernen müssen, womit sie sich offenbar nicht abfinden wollen?" (FR 15.10.96, S. 25). Dem Protest der 
Schriftsteller wird der Anstrich einer ,ewigen Nörgelei“ vorgeworfen (Die Welt, 11.10.96).

Lutz Götze schreibt in der Süddeutschen Zeitung vom 5.11.96:

„Nun ist einer aus dem Tiefschlaf erwacht und zieht mit missionarischem Eifer, von Weilheim aus, durch die Lande, um diesem 'Angriff auf die deutschsprachige Literatur' den Garaus zu machen. Die Dichterschar springt auf den vermeintlich fahrenden Zug, greift zum Inventar der Rüpelprosa ('Sesselfurzer' des Hans Magnus Enzensberger) statt des einstmals gepflegten Stils, in der mittelfränkischen Provinz wird sogleich sekundiert (Ickler), der das 'Rechtschreibchaos' am Horizont ausmacht, und der Präsident des Goethe-Instituts verweist auf den 'Kulturverfall', der den Deutschen bevorstehe. Ganz Aufgeregte wollen die Rechtschreibreform gar zum Unwort des Jahres küren. Rauschhaftes Gerede auf Stammtischniveau!“

Der letzte Satz ist noch in einer anderen Hinsicht aufschlußreich. Kennzeichen öffentlich geführter Debatten in Deutschland ist es häufig, die Sprachhaltung oder die Argumentationslinien des ausgemachten Gegners zu kommentieren. In einem Leserbrief aus der gleichen Ausgabe, der die „Argumentationslinien“ der Gegner der Reform darstellt - sie sei erstens inkonsequent, zweitens zu teuer -, heißt es: „Das Wesen beider Argumentationslinien ist letztendlich die Ablehnung des Neuen allein deshalb, weil es (in diesem Falle eher geringfügig) anders ist als das bisher Gewohnte." (SZ 5.11.96)

Im Tagesspiegel vom 23.12.96 wird geschrieben: „Ich denke, daß eine solche Auseinandersetzung deshalb besonders wichtig war und noch ist, weil offensichtlich nicht nur diese unsäglichen deutschen Besserwisser und Oberlehrer vom Schlage eines Herm Denk wieder einmal 'Verrat am Vaterland' wittern ..., sondern offensichtlich auch die sogenannten literarischen Eliten sich von ihrer gesellschaftlichen Verantwortung verabschiedet haben".

Dem Schreiber ist sicher nicht bewußt, daß er einerseits selber damit Klischees transportiert, die auf prototypische deutsche Nationaleigenschaften gehen, und zum andern eine ganz bestimmte, nun auch sehr deutsche Vorstellung von den "Aufgaben" der Schriftsteller hat.

Wie bei dem Vorwurf an die Gegner, ihr Protest käme zu spät, hier habe der deutsche Michel wieder einmal - wie bei jeder Reform - geschlafen, wird auch bei der Argumentation, der Protest sei völlig übertrieben, ins Grundsätzliche gegangen. Sachargumente finden sich dann immer weniger. Der Streit wird benutzt, die Gegner als anmaßend, konfus und lächerlich darzustellen.

„Anstatt sich darüber zu freuen, daß es künftig statt zweihundert nur noch hundert Rechtschreibregeln zu beachten gilt, wird gegen diese Reform angegangen, als gelte es, das christliche Abendland vor dem Untergang zu bewahren." (StZ 11.3.97) 
Der folgende Schreiber merkt nicht, daß er selber ,seinen Senf dazugibt“, wenn er schreibt: „Nun hat das einfältige Gemecker über die Rechtschreibreform auch den Bundestag erreicht. Und natürlich darf in diesem unserem Lande jeder seinen Senf dazugeben." (SZ 5.5.97)

Es fehlt allerdings auch nicht an Versuchen, die Diskussion von ihren Verstiegenheiten herunterzuholen. So heißt es in einem Leserbrief in der Frankfurter Allgemeinen Zeitung vom 20.1.97 zunächst ironisch: „Die Schieflage der Reform macht die Grenzen der Staatsmacht deutlich. Das Volk erobert sich seine Sprache zurück. 'Wir sind das Volk'“ und dann: „Von dem verstorbenen Professor Peter Brückner aus Hannover stammt der schöne Satz, die korrekte Rechtschreibung sei das Statussymbol des kleinen Mittelstandes. Oberhalb von dieser Ebene hat man die Orthographie noch nie sonderlich ernst genommen."

\subsection{Die Kommentare}

In dem von mir untersuchten Zeitraum fanden sich 420 Kommentare zur Rechtschreibreform, auch dies eine, verglichen mit anderen kulturpolitischen Themen, außerordentlich große Zahl. Das Verhältnis Befürworter: Gegner ist hier zwar nicht so deutlich wie bei den Leserbriefen, es überwiegen aber die Gegner auch hier. Doch - und dies ist sehr wichtig: Sie liegen unterhalb der 50\%-Grenze. Genauso begegnen auch hier so gut wie alle aus den Leserbriefen bekannten Argumente und Argumentationsschemata, auf die gleich genauer einzugehen ist. Was besonders auffällt, ist, daß im fast genau gleichen Maße wie bei den Leserbriefen die Kommentare eindeutig Stellung nehmen, wirklich neutrale gibt es nur äußerst selten. Als berufsmäßige Schreiber haben die Journalisten ein viel engeres und damit engagierteres Verhältnis zu Sprache und Schrift. Dazu kommt, $\mathrm{da} \beta$ die Redaktionen ziemlich frühzeitig festgelegt sind, und zwar ebenfalls polar, allerdings mit graduellen Unterschieden. Von den großen Tages- und Wochenzeitungen kommentieren Die Welt so gut wie ausschließlich, die Frankfurter Allgemeine Zeitung und Der Spiegel ganz überwiegend ablehnend, die Frankfurter Rundschau, das Neue Deutschland und Die Zeit fast nur zustimmend, ganz gleich, welches Faktum neu in die Diskussion kommt. Die Süddeutsche Zeitung ist bemüht, den Reformimpetus zu würdigen, wird aber zunehmend skeptischer in der Kommentierung.

Insgesamt ist jedenfalls auch in den Kommentaren nicht zu verkennen, $\mathrm{da} ß$ eine Polarisierung eingetreten ist. Vorsicht ist allerdings geboten, wenn hiermit eine Korrelation zum Image der Zeitungen als konservativ, progressiv oder liberal gesucht wird. Wie eingangs betont wurde: die Rechtschreibdebatte ist ein Beispiel für eine quasipolitische Diskussion: Sie wird öffentlich geführt, sie ist polemisch, sie geht ins Grundsätzliche. Aber da sie durch die Exekutive. in Deutschland durch die der Kultusmi- 
nister, vollkommen einheitlich vertreten wird und damit keinen politisch, etwa durch Parteitraditionen geprägten Ausdruck mehr finden kann, brechen die gegensätzlichen Positionen sich heterogen Bahn. Dies soll nicht heißen, daß 'Bewahrer' und 'Fortschrittler' nicht aufeinanderstießen. Im Gegenteil, der Vorwurf, sich dem Fortschritt zu verweigern, trifft vehement auf die Gegenposition, sich an der Tradition zu vergreifen, und dies häufig grundsätzlich. Dafür einige wenige Beispiele: „Eine lebendige Sprache befindet sich in einem ständigen Reformationsprozeß, an dem alle, die in ihr sprechen und schreiben, ihren Anteil haben. Und sie wächst dabei immer wieder aus dem Korsett der Orthographie heraus und macht damit neue Rechtschreibregeln notwendig, um sie nicht in den alten ersticken zu lassen." Die Rechtschreibreformer hätten dies allerdings nicht plausibel machen können. (Ulrich Baron im Rheinischen Merkur 31.1.97). In der Leipziger Volkszeitung findet sich am 19.8.97 ein Kommentar mit dem Titel „Alles ist im Fluss“. (Die Reformgegner argumentieren entweder mit dem Bruch der Tradition oder führen einzelne sachliche Bereiche an, bei denen nach ihrer Meinung die Neuregelung in Bewährtes eingreife (z. B. Horst Haider Munske, Tsp 5.7.97). Bisweilen geht es dabei - auch in der Kommentierung - ins Grundsätzliche: „Man merkt eine ideologisch-erzieherische Absicht und ist verstimmt." (Daniela Stigl, Lit. u. Kritik, 31.10.96). Besonders vehement äußert sich Adolf Muschg in der Weltwoche 17.10.96: „Man muß aufwachen, wenn Sprache und Regelung zusammengehen." Ob dieses grundsätzliche Mißtrauen hier angebracht ist, muß bezweifelt werden. $\mathrm{Da} \beta$ der Staat hier eine Regelungskompetenz hat, wird im Zuge der Kommentierung der Gerichtsurteile durchaus und mehrfach anerkannt (Tsp 10.11.95), andererseits, vor allem durch den Verfassungsrechtler Ernst Gottfried Mahrenholz, auch grundsätzlich bestritten: „Nicht der Staat verfügt über die Sprache.“ (SZ 23.8.97). Gegen den obrigkeitlichen Eingriff in die Kompetenz der Bürger wird auch in den Kommentaren zu Felde gezogen. Es wird von der „unseligen Regelungswut der Bildungspolitiker gesprochen“ (Peter M. Mack, Main-Post 30.7.97). Die Reform sei das Ergebnis der Wichtigtuerei einiger Experten (Barbara Petsch, Die Presse 11.10.96).

Hauptargumente der Befürworter gegen die Reformgegner sind erstens: Der Protest kommt zu spät. Die Leute haben geschlafen. - Zweitens: Die Gegner sind grundsätzlich reformunwillig. Der Verspätungsvorwurf bricht vor allem nach der 'Frankfurter Erklärung' auf. Dieser Name wird im übrigen auch einmal als übertrieben pathetische Wortwahl kritisiert (Tsp 8.10.976). Es wird von „Klassenkampf gegen die Reform“, mit Anspielung auf den Gymnasiallehrer Denk gesprochen, der ein apokalyptisches Szenario entwerfe (Michael Braun, BZ 9.10.96). Der Aufstand der Dichter sei reaktionär (Joachim Worthman, StZ 9.10.96), Hermann Unterstöger fragt in der Süddeutschen Zeitung vom 12./13.10.96, „Wo waren 
die Dichter in den letzten Jahren?", die Formulierung „Ein Wolfsgeheule post factum" (Die Zeit 11.10.96) ist wesentlich polemischer. Vom Amoklauf der Autoren spricht Roger Köppel (TA 15.10.96). „Die Gegner wurden erst nach Ladenschluß so richtig wach" (Die Zeit 1.8.97), aber schon vorher hatte Thomas Steinfeld in der Frankfurter Allgemeinen Zeitung (24.10.96) geschrieben: „Für Argumente gelten keine Ladenschlußzeiten."

Was nach der eingetretenen Polarisierung die Markierung und Bekämpfung der gegnerischen Position betrifft, so finden sich hier fast noch schärfere Töne als in den Leserbriefen. So sprachen am gleichen Tag (8.8.97) Die Welt von der „vom deutschen Provinzialismus autoritär diktierten Rechtschreibänderung " (Lothar Rühl) und Die Zeit davon, daß das Schlimmste an der Rechtschreibreformdebatte ,ihre passionierten Gegner" (Jörg Lau) seien. Die Besprechung von Horst Haider Munskes Buch "Orthographie als Sprachkultur" in der Frankfurter Allgemeinen Zeitung (vom 22.5.97) wird dort mit der Überschrift versehen: „Kollege mit Abrißbirne“, Untertitel: „Wie bei der Mafia: Aus dem inneren Zirkel der Rechtschreibreformer". Die Frankfurter Rundschau (8.10.96) hält dagegen den Gegnern „schwammigen Kulturkonservatismus" und „weinerliche Gralshüter-Gebärde“ vor und mokiert sich über „die grotesken rhetorischen Hyperbeln, die schiefen Metaphern, das trübe Gemisch aus moraliner Empörung, ästhetischem Ekel und wohlfeilen Protestformeln“. Dieter E. Zimmer listet die seiner Meinung nach ,vorwiegend irrationalen Gründe" der Gegner auf (Die Zeit 15.8.97):

„1. die Reform sei im Hauruckverfahren durchgepeitscht worden. - Das läßt sich leicht widerlegen.

2. Die Rechtschreibung sei das in Jahrhunderten herangereifte Werk einer ganzen Kulturgemeinschaft, etwas Heiliges geradezu, an dem keiner herumkurieren dürfe. - Auch dies ist mit Sicherheit eine übertriebene Position.

3. Der Eingriff in die Rechtschreibung sei ein Eingriff in 'die Sprache' selber.

4. Dem Volk und sogar seinen Schriftstellern würden allerlei unerwünschte Schreibungen aufgezwungen. - Irrtum. Die Orthographie gilt für Schulen und Behörden. Sonst hat jeder die Prärogative, zu schreiben, wie er will."

Das nun wieder ist eine sicher etwas idealistische Position. Wer wollte bestreiten, daß auf die Dauer die Normsetzungen in der Schule nicht für alle verbindlich würden. Und der Schlußpassus aus Zimmers Artikel spricht dies auch indirekt an: ,Jemand muß Zweifelsfälle entscheiden und die Regeln wenn nötig an neue Sprachsituationen anpassen, und allen gefallen werden diese Regelungen nie.“ 
In der Tat darf dieser Gesichtspunkt nicht außer acht gelassen werden. Die Regelung von 1901 und die Entwicklung, die dahin geführt hatte, war von dem Willen getragen, die Einheitlichkeit als oberstes Prinzip der Rechtschreibung zu akzeptieren. Die seinerzeit vorfindlichen realen Divergenzen wurden dadurch im Regelungsweg beseitigt, wissenschaftlich begründbare Alternativen mußten zurückstehen. Seit dieser Entscheidung ist das Prinzip der Rechtschreibregelung in Kraft, und es denkt auch niemand ernsthaft daran, es grundsätzlich aufzuheben. Die Konsequenz daraus ist, daß staatliche Kompetenz in die Rechtschreibung eingegangen ist, die Schreibung ist seitdem nicht mehr naturwüchsig. Daraus ergibt sich auch, daß sie heute notgedrungen einen fixierten Stand, nämlich den des im 19. Jahrhundert erreichten aufweist. Ansatz der Rechtschreibkommissionen war es, über diesen Stand hinaus zu gelangen. Ohne Einzelheiten der Reform hier zu kommentieren, kann aber gesagt werden, daß vor allem wohl aus diesem Grund bei den Reformbefürwortern eine ablehnende Haltung Reformen gegenüber auf Unverständnis stoßen mußte. Den Reformern drängt sich auf, in den Gegnern der Reform allgemeine Verweigerer zu sehen. So wird im Sonntagsblatt geschrieben: „Jetzt reiben sie sich wieder die Hände“. Die Deutschen seien eben eine Nation der Blokkierer (ao 1.8.97). In der Frankfurter Rundschau heißt es: „Schlechte Zeiten für Reformen in Deutschland. Stillstand wohin man blickt." (AH 30.7.97). Im Tagesanzeiger (Martin Halter 25.7.97) wird sogar von einer „außerparlamentarischen Opposition" gesprochen. Besonders im Zusammenhang mit der Anrufung der Gerichte schlägt sich die Auffassung nieder, daß das Volk reformunwillig sei. In der Frankfurter Allgemeinen Zeitung (Eckhard Fuhr 12.8.97) heißt es: „Die Verhinderungsmacht der Teilgewalten lähmt das Land" und in der Leipziger Volkszeitung (Armin Grötz 14.8.97): „So hofft diesmal das reformunwillige Volk auf die letzte Instanz."

Es läßt sich nicht verleugnen, daß der Reformstau - nicht zufällig ist dieser Ausdruck das 'Wort des Jahres 1997' - neben der Steuer-, der Renten-, der Gesundheitsreform und anderen Reformvorhaben auch die Rechtschreibreform betrifft. Dies wird gern in Karikaturen aufgegriffen und mir scheint, daß die Zeichner ganz realistisch doch den Relevanzunterschied markieren.

Zwar ist, wie inzwischen mehrfach festgestellt worden ist, die Rechtschreibreform ein quasi politisches Debattenfeld ${ }^{8}$, aber eben doch nicht

8 Helmut Berschin urteilt folgendermaßen: „Die Rechtschreibreform ist ein politisches Projekt auf wissenschaftlicher, genauer: linguistischer Grundlage. “ (Berschin 1998, S. 48). Dieses Projekt habe nicht glücken können, „weil es unter einem politischen Vorzeichen stand: dem 'Kompromiß'“. Kompromisse seien politische, nicht aber wissenschaftliche Konsensfindungskategorien. 


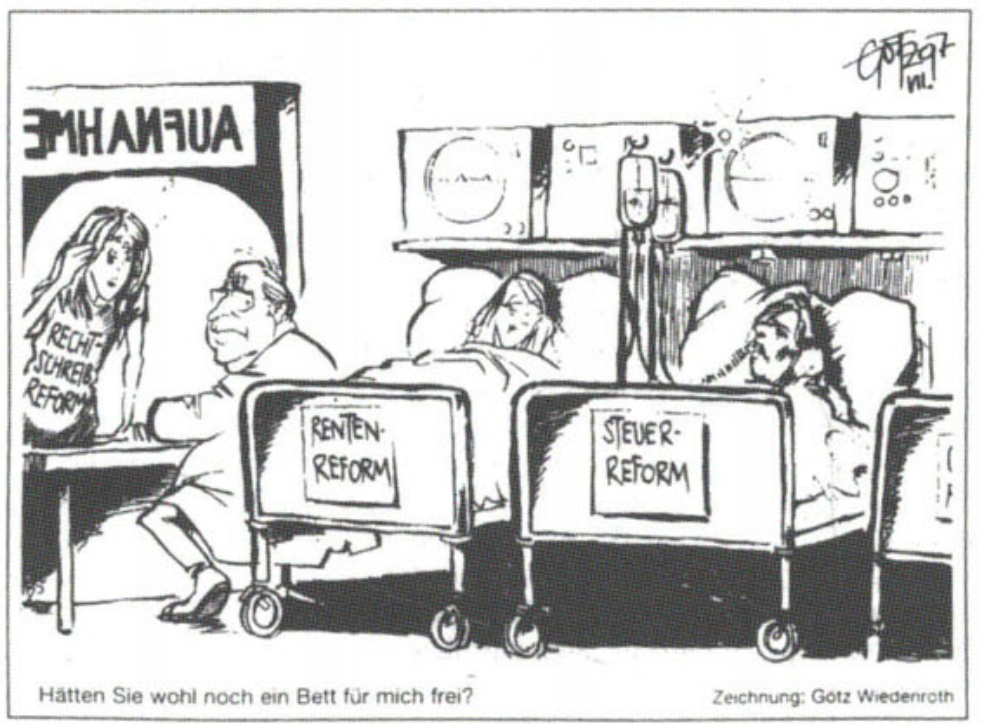

eines, das den demokratischen Instanzenweg durchlaufen hat. Vielleicht sind die Aktivitäten der Gegner unter dem Aspekt zu sehen, diesen Weg im Nachhinein noch zu ermöglichen: durch Pochen auf parlamentarische Behandlung, durch Anrufung der Gerichte und mit der Vorbereitung von Volksbegehren. Es ist allerdings schlecht vorstellbar, daß die höchstrichterliche Instanz der Rechtschreibung ein solches Gewicht beilegen wird. Die Kommentare in der Presse sind, im Vergleich mit den anderen Aspekten der Diskussion um die Rechtschreibreform, hier auch ausgesprochen vorsichtig.

Was den Vergleich mit den anderen anstehenden Reformen betrifft, so ist, abgesehen von der Relevanz noch auf einen Verfahrensunterschied hinzuweisen: Bei der Steuerreform, der Gesundheitsreform und Rentenreform handelt es sich um innenpolitische Thematiken. Sie sind gänzlich auf die Bundesrepublik beschränkt. Bei der Rechtschreibreform liegt ein staatenübergreifendes Themenfeld vor. Bei einem Vergleich müssen natürlich diese Prämissen beachtet werden. D. E. Zimmer in der Zeit (14.11.97) ist allerdings der Meinung, daß die Rechtschreibung gar den Euro vertreten muß. Wenn man auf die Vehemenz der Argumentation schaut, trifft dies wohl zu. Die Relevanzen sind dann aber doch wohl unterschiedliche.

Im übrigen ist es das gute Recht der Bürger, alle Wege zu versuchen, Entscheidungen, die sie nicht billigen, anzufechten, sei es nun auf dem Ge- 
richtswege oder sei es durch das Institut des Volksbegehrens. Was das erstere betrifft, so zeigen die bislang vorliegenden Entscheidungen in ihrer Unterschiedlichkeit, daß zumindest ein rechtlich kontrovers beurteiltes Phänomen vorliegt. Über den Status der Rechtschreibung werden wir nach Abschluß der juristischen Verfahren jedenfalls besser Bescheid wissen. Die Rechtschreibung ist sicher nicht ein bloß formales Regelungsinstrument wie die Verkehrszeichen oder die Postleitzahlen, wo dem Staat die Regelungskompetenz niemand ernsthaft streitig machen würde. Sie ist aber auch sicher nicht ein unverwechselbares individuelles Persönlichkeitsmerkmal. Wenn Schriftsteller wie Arno Schmidt und Ernst Jandl ihre private Rechtschreibung kreieren, dann wirkt dies eben nur auf dem Hintergrund einer akzeptierten Norm. Ob die Norm in ihrer Regelung Bandbreiten der Verbindlichkeiten zuläßt oder nicht, ist eine Sache der Konsensfindung.

In bezug auf die initiierten Volksbegehren ließe sich schließlich anmerken, daß dies ein weiterer Schritt dazu ist, den legitimierten Volksvertretern ihre Kompetenzen aus der Hand zu nehmen. Wer aber vertritt die Bevölkerung in orthographischen Fragen? Schon diese Fragestellung weist der Sache auf den ersten Blick übertriebene Bedeutung zu. Sie erklärt aber vielleicht doch, daß wir im Zuge der Rechtschreibdebatte eine Hinwendung zu Autoritäten zu beobachten hatten, die ganz erstaunlich sind. Vielleicht wird die Meinung der Schriftsteller als stellvertretend in Bezug auf dieses Thema, jedenfalls als besonders kompetent empfunden. Zu erklären wäre aber immer noch, warum die Schriftsteller und die anderen Autoritäten, die sich geäußert haben, so überwiegend negativ reagiert haben. Wenn sie sich hier in ihrer Stellvertreterfunktion begreifen, dann ließe sich die Erklärung darin finden, daß sie sich übergangen fühlen.

\section{Resümee}

Die zuletzt angestellten Überlegungen lassen sich weiter verallgemeinern: In der Frage der Rechtschreibung werden normsetzende oder gar normändernde Autoritäten schlecht akzeptiert. Dies ist auch nicht anders zu erwarten. Zwar ist der rechtliche Status der Orthographie nicht gänzlich geklärt, de facto ist sie aber mehr als eine Verbindlichkeitsempfehlung, allerdings auch weniger als eine etwa gesetzlich gegründete „Schreibverkehrsordnung".

Die gegenwärtig noch gültige Regelung von 1901 ist durch den Willen zur Einigung zustande gekommen; von Orthographietheoretikern vorgeschlagene Alternativen waren zurückgetreten. Die jetzt geplante Änderung ist ebenfalls eine Regelung, in der Kompromisse vorgesehen sind.

Die Debatte über die Rechtschreibung wird öffentlich geführt. Es wäre unangebracht, sich abfällig über die z. T. krause Metaphorik, die unbeholfene Argumentationstechnik oder die Schärfe der Argumentation zu äu- 
ßern. Letzteres ist eine Konsequenz, die sich bei politischen Debatten und auch solchen, die einen quasipolitischen Status haben, nicht vermeiden läßt. Die dahinter zu vermutende allgemeine Ratio ist, daß alles öffentlich zur Sprache gebracht wird, was mit dieser Thematik zusammenhängt, auch mit der Konsequenz, daß die Fachleute in dieser Auseinandersetzung nicht mehr den Ton angeben. Auch daß die öffentliche Meinung zu einer Polarisierung führt, läßt sich kaum vermeiden.

Wie alle öffentlichen Themen wird auch die Rechtschreibdebatte instrumentalisiert, einmal dadurch, daß die Thematik direkt politisch in Anspruch genommen wird, wie es bei einigen Politikern zu sehen war, zum anderen dadurch, daß Autoritäten sich äußern, die von der Schreibung in besonderem Maße betroffen sind, schließlich, daß sie als Aufhänger benutzt wird, um über den Zustand der deutschen Sprache öffentlich zu lamentieren.

Zu weitgehende Schlüsse sollte man aus der Rechtschreibdebatte jedoch nicht ziehen. Die Analogie zu den Diskussionen um die anstehenden Reformen im sozialen und politischen Gefüge sind zu hoch gegriffen. Man könnte eher sagen: Nicht anstelle der Steuer- oder Gesundheitsreform tobt der Kampf nun in der Rechtschreibreform, sondern: die Bürgerinnen und Bürger engagieren sich, wie zu sehen, auch bei einem kulturpolitischen Thema.

Im Interesse der Sache ist jetzt natürlich ein Ende der Debatte zu wünschen. Bedauerlich, wenn auch unvermeidlich, ist es wohl, daß in Deutschland dies auf dem Gerichtsweg erreicht werden muß. Dies ist jedoch immer noch besser als eine bloß exekutive Regelung, die wie zu sehen war, nur widerstrebend akzeptiert wird.

Die Linguisten sollten sich allerdings aufgerufen fühlen, der Bevölkerung Antworten auf ihre Fragen zu geben, auch wenn es mühsam sein sollte, sie hinter den starken Emotionen erst freizulegen.

Zitate aus folgenden Tages- und Wochenzeitungen:

Basler Zeitung (BZ)

Das Sonntagsblatt (DS) (Deutsches Allgemeines Sonntagsblatt (DAS))

Der Spiegel

Der Standard

Der Tagesspiegel (Tsp)

Die Furche

Die Presse

Die Tageszeitung (taz)

Die Welt

Die Woche

Die Zeit

Dolomiten (Dol)

Erlanger Nachrichten

Frankfurter Allgemeine Zeitung (FAZ) 
Frankfurter Rundschau (FR)

Gegenwart

Leipziger Volkszeitung (LVZ)

Literatur und Kritik (Lit. u. Kritik)

Main-Post

Mittelbayerische Zeitung (MZ)

Münchner Merkur (MM)

Neues Deutschland (ND)

Neue Zürcher Zeitung (Fernausgabe) (NZZ)

Oberösterreichische Nachrichten (OÖN)

Passauer Neue Presse (PNP)

Rheinischer Merkur (RM)

Salzburger Nachrichten (SN)

Stuttgarter Zeitung (StZ)

Süddeutsche Zeitung (SZ)

Südtiroler Illustrierte (FF)

Tages Anzeiger (TA)

Weltwoche (WW)

Westdeutsche Allgemeine Zeitung (WAZ)

Westfälische Rundschau (Westf. Rundschau)

\section{Literatur}

Augst, Gerhard/Blüml, Karl/Nerius, Dieter/Sitta, Horst (Hg.) (1997): Zur Neuregelung der deutschen Orthographie. Tübingen.

Berschin, Helmut (1998): Laufenlassen oder nicht laufen lassen? Eine Zwischenbilanz zur Rechtschreibreform. In: Zeitschrift für Dialektologie und Linguistik 55, S. 42-49.

Eisenberg, Peter (1995): Die deutsche Sprache und die Reform ihrer Orthographie. In: Praxis Deutsch 130, S. 3-6.

Eroms, Hans-Werner (1997): Die Gewichtung des 'historischen Prinzips' in der deutschen Orthographie. In: Glaser, Elvira/Schlaefer, Michael (Hg.): Grammatica ianua artium. Festschrift für Rolf Bergmann zum 60. Geburtstag. Heidelberg, S. 221-235.

Eroms, Hans-Werner/Munske, Horst Haider (Hg.) (1997): Die Rechtschreibreform. Pro und Kontra. Berlin.

Gallmann, Peter/Sitta, Horst (1996): Die Neuregelung der deutschen Rechtschreibung. Regeln, Kommentare und Verzeichnis wichtiger Neuschreibungen. Mannheim/Leipzig/Wien/Zürich (Duden-Taschenbücher 26).

Internationaler Arbeitskreis für Orthographie (Hg.) (1992): Deutsche Rechtschreibung. Vorschläge zu ihrer Neuregelung. Tübingen.

Jung, Matthias/Wengeler, Martin (1998): Wörter - Argumente - Diskurse. Was die Öffentlichkeit bewegt und was die Linguistik dazu sagen kann. In diesem Band.

Maas, Utz (1992): Grundzüge der deutschen Orthographie. Tübingen (Reihe Germanistische Linguistik 120). 
Munske, Horst Haider (1997): Orthographie als Sprachkultur. Frankfurt.

Nerius, Dieter (1997): Was soll und was kann eine Orthographiereform leisten? In: Eroms/Munske (1997), S. 157-163.

Nerius, Dieter et al. (1989): Deutsche Orthographie. 2. Aufl. Leipzig.

Piel, Edgar (1997): Fast niemand will die Rechtschreibreform. Bericht über eine demoskopische Sondierung. In: Eroms/Munske (Hg.) (1997), S. 165-172.

Rechtschreibreform (1996). Eine Zusammenfassung von Dr. Klaus Heller. In: Sprachreport Extra Ausgabe Juli 1996.

Scheuringer, Hermann (1997): Auf den Schlips getreten: Zur Perpetuierung einer öffentlichen Erregung. In: Eroms/Munske (1997), S. 197-204.

Zabel, Hermann (Hg.) (1989): Der gekippte Keiser. Bochum.

Zemb, Jean-Marie (1997): Für eine sinnige Rechtschreibung. Eine Aufforderung zur Besinnung ohne Gesichtsverlust. Tübingen. 